\title{
Developing a Patient-Centered Community-Based Model for Management of Multi-Drug Resistant Tuberculosis in Uganda: A Mixed Methods Study
}

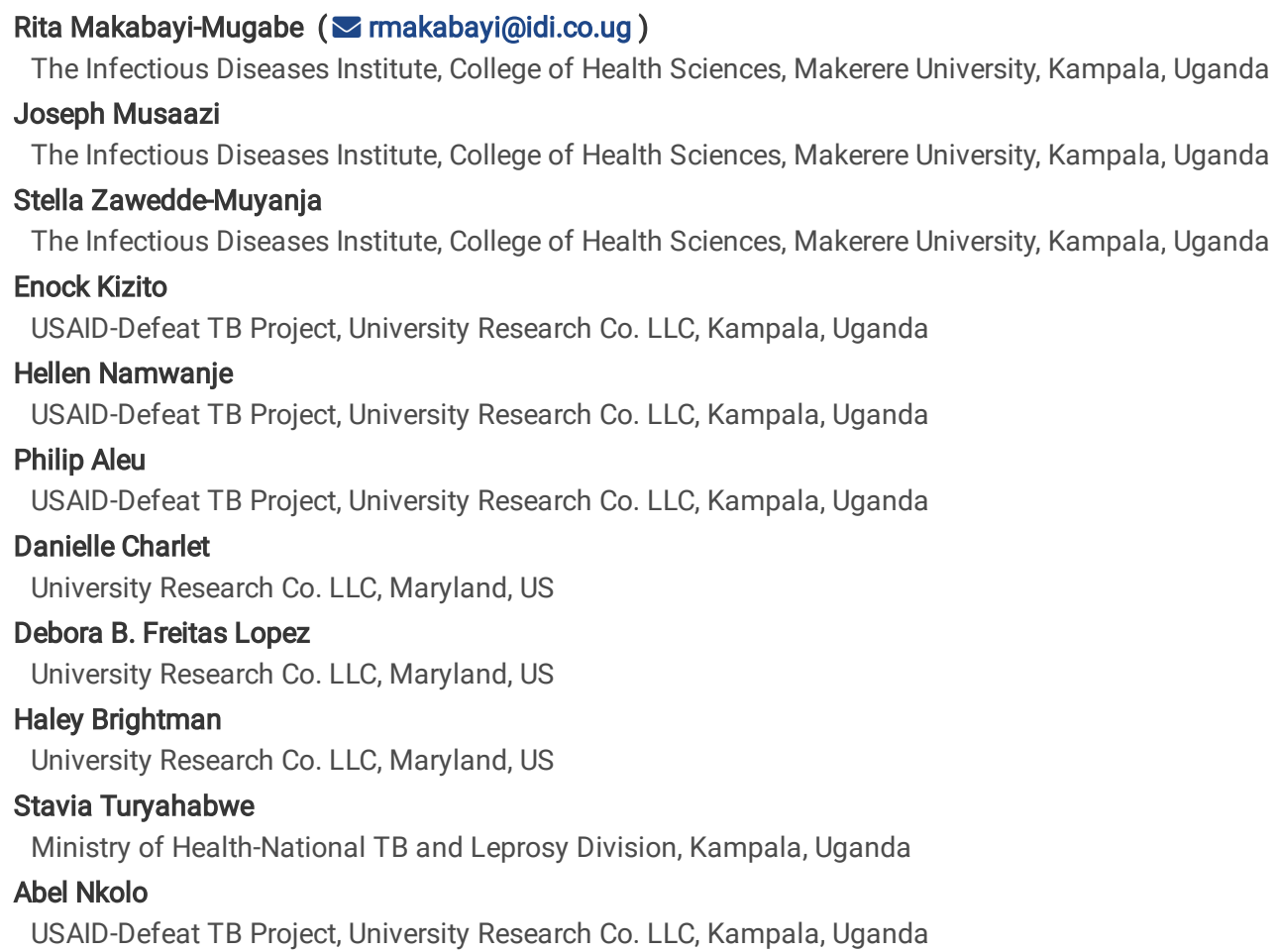

\section{Research Article}

Keywords: Community-based care, Directly Observed Therapy, Multi-drug Resistant Tuberculosis, Uganda, Discrete Choice Experiment

Posted Date: April 30th, 2021

DOI: https://doi.org/10.21203/rs.3.rs-442837/v1

License: (a) (i) This work is licensed under a Creative Commons Attribution 4.0 International License. Read Full License 


\section{Abstract}

\section{Background:}

The advent of all-oral regimens for the management of multi-drug resistant tuberculosis (MDR-TB) makes the implementation of community-based directly observed therapy (CB-DOT) a possibility for this group of patients. We set out to determine patient preferences for different attributes of a community-based model for the management of MDR-TB in Uganda.

\section{Methods:}

The study was conducted at five tertiary referral hospitals. We used a parallel convergent mixed methods study design. To collect quantitative data, we conducted a discrete choice experiment (DCE) with three different attributes of community-based care (provider type, location of care, and type of support) combined into eight choice sets, each with two options and an opt-out. We collected additional qualitative data by eliciting patient reasons for selection of each choice set. We fitted a mixed logit choice model to determine patient preferences for different attributes of community-based care. Thematic analysis using NVivo12 was done to understand the reasons for the choices made.

\section{Results:}

From December 2019 to January 2020, we interviewed 103 patients with MDR-TB. Majority (58.3\%) were male; $61.2 \%$ were HIV negative; and the median age was 37 (IQR 30-47) years. Two thirds (65.1\%) earned less than \$1 per day. Study participants preferred at least one of the CB-DOT models of care to none (current standard of care). The most preferred model consisted of a community health worker (CHW) giving DOT at home and travel vouchers to enable attendance at monthly clinic follow-up visits. Qualitative interviews revealed that patients perceived CHWs as knowledgeable and able to offer psychosocial support. Patients also indicated a preference for taking medication at home because it saves both time and money and presents a lower risk of being stigmatized.

\section{Conclusion:}

People with MDR-TB prefer to be supported to take their medicine at home by a member of their community. The effectiveness of this model of care will be further evaluated.

\section{Background}

Multi-drug resistant tuberculosis (MDR-TB), defined as resistance to both isoniazid and rifampicin, the two major first line anti-TB medicines, threatens global TB control efforts and remains a major public health concern in many countries. ${ }^{1}$ Globally, case detection and treatment success rates for MDR-TB are suboptimal. In 2019 , only $57 \%$ of patients started on second line therapy were successfully treated. ${ }^{2}$ In Uganda, $64 \%$ of those started on treatment in 2016 were successfully treated while an estimated $19 \%$ died and $15 \%$ were lost to follow up. ${ }^{3}$ These suboptimal treatment outcomes are a potential risk for the development and spread of further resistance to TB treatment. ${ }^{4}$

Uganda currently implements a mixed model of care for MDR-TB characterized by initial hospitalization for two to eight weeks followed by ambulatory directly observed therapy at a public or private health facility near the patient's home. ${ }^{5}$ However, delivery of care through a health facility-based directly observed therapy (HF-DOT) model has been documented to significantly contribute to poor treatment outcomes particularly in resource limited settings ${ }^{6}$. Patients who receive care through this model experience various inconveniences (e.g., travel and waiting times) and incur significant direct (e.g., transport costs) and indirect costs (e.g., time lost from work) that hinder successful treatment completion. ${ }^{6}$ In the management of both drug susceptible and drug resistant TB, community-based treatment support models have been associated with improved treatment outcomes and increased cost effectiveness compared to health facility-based models. ${ }^{7,8,9,10}$

Since March 2018, the shorter 9-12 months treatment regimen has been the standard of care for patients with MDR-TB without extrapulmonary TB or resistance to fluoroquinolones or second line injectable drugs amongst others. This shorter treatment regimen is better tolerated, and in clinical trials resulted in better treatment outcomes than the two-year treatment regimen. ${ }^{11}$ However, this regimen also has lower tolerance for lapses in adherence and there is only one chance to complete the shorter regimen. ${ }^{5}$ Provision of effective adherence support for patients receiving the shorter MDR-TB regimen is therefore a priority intervention for the National TB and Leprosy Program (NTLP). Further, the use of community health workers (CHWs) to offer treatment adherence support, particularly in the continuation phase of treatment, is recommended in the Uganda National Guidelines for Programmatic Management of Drug Resistant Tuberculosis ${ }^{5}$ but is yet to be implemented. We therefore set out to assess patient preferences for different attributes of community-based care for MDR-TB.

\section{Methods}

\section{Study setting}

The study was carried out at five tertiary referral hospitals purposively selected because they provide care to about $80 \%$ of patients started on second line TB treatment in Uganda every year. Second line TB treatment consists of six drugs; kanamycin (Km), moxifloxacin (Mfx), Ethionamide (Eto), clofazimine $(\mathrm{Cfz})$, pyrazinamide $(\mathrm{Z})$, high dose Isoniazid $(\mathrm{H})$ and ethambutol $(\mathrm{E})$ given in two phases; an intensive four-to-six-month period and a five-month 
continuation phase. In the initial intensive phase, all seven drugs are given while in the continuation phase only four drugs are given that include Mfx, Cfz, Z and $E^{5}$

\section{Study Design}

We used a parallel convergent mixed methods study design to collect both quantitative and qualitative data on patient preferences for different attributes of community-based care.

\section{Study Variables}

The dependent variable was the preferred community-based directly observed therapy (CB-DOT) model, which was the most common chosen combination of the three different CB-DOT attributes that included principal care giver, location of care, and type of additional support. Independent variables included patient demographics, e.g., age, sex, education, duration on MDR-TB treatment, and HIV status.

\section{Data Collection}

We included patients 18 years or older who had completed the intensive phase of treatment for MDR-TB. We used stratified sampling to determine the number of patients to be selected from each hospital, and simple random sampling to select the patients to be interviewed at each hospital. We collected quantitative data on patient preferences using a discrete choice experiment (DCE), a technique for eliciting individual preferences for a product or

service. ${ }^{12}$ We chose three attributes (provider type, location of service, and provision of additional support) each with two or three attribute levels (Table 1 ). Attributes used in this study were chosen from an initial exploratory study carried out six months prior. Using a fractional factorial design, we chose eight choice sets. Recommendations in the literature ${ }^{13,14}$ have shown that more than eight choice tasks are a cognitive and time burden for participants. We included an opt-out response category so that respondents could choose "neither" choice set to reflect dissatisfaction with either potential CB-DOT model. The final design had $96 \%$ d-efficiency (Table 2). To cater for limited literacy levels among the respondents, visual representations of each attribute were developed and used during data collection. Data was collected electronically and entered directly into TB Info, a web-based system.

Additional quantitative data on age, gender, marital status, date of treatment initiation, duration on MDR-TB treatment, and underlying co-morbidities was abstracted from health facility records. Qualitative data was collected through patient interviews carried out after each choice set was presented to find out the reasons for choices made for each set of attributes.

Table 1

DCE attributes and levels

\begin{tabular}{|ll|}
\hline Attribute & Level \\
\hline Provider type & Expert client \\
& Family member \\
\cline { 2 - 2 } Location & Community health worker \\
\cline { 2 - 2 } Support type & Some reminders \\
& Call reminders \\
& Travel vouchers \\
\hline
\end{tabular}


Table 2

DCE choice sets each with an opt out option.

\begin{tabular}{|c|c|c|c|c|}
\hline \multirow[t]{2}{*}{ Choice Set } & \multirow[t]{2}{*}{ Choice set alternatives } & \multicolumn{3}{|l|}{ Attributes } \\
\hline & & Location of care & Provider type & Support type \\
\hline \multirow[t]{3}{*}{1} & 1 & work & $\mathrm{CHW}$ & Travel vouchers \\
\hline & II & Home & Expert Client & Call reminders \\
\hline & III & Neither & & \\
\hline \multirow[t]{3}{*}{2} & 1 & Home & Family member & SMS reminders \\
\hline & II & work & Expert Client & Travel vouchers \\
\hline & III & Neither & & \\
\hline \multirow[t]{3}{*}{3} & 1 & Home & Family member & SMS reminders \\
\hline & II & work & $\mathrm{CHW}$ & Call reminders \\
\hline & III & Neither & & \\
\hline \multirow[t]{3}{*}{4} & 1 & Home & Expert Client & SMS reminders \\
\hline & II & Home & Family member & Call reminders \\
\hline & III & Neither & & \\
\hline \multirow[t]{3}{*}{5} & 1 & Home & Family member & Travel vouchers \\
\hline & II & Home & $\mathrm{CHW}$ & SMS reminders \\
\hline & III & Neither & & \\
\hline \multirow[t]{3}{*}{6} & 1 & Work & $\mathrm{CHW}$ & SMS reminders \\
\hline & II & Home & Expert Client & Travel vouchers \\
\hline & III & Neither & & \\
\hline \multirow[t]{3}{*}{7} & 1 & Work & Expert Client & SMS reminders \\
\hline & II & Home & $\mathrm{CHW}$ & Call reminders \\
\hline & III & Neither & & \\
\hline \multirow[t]{3}{*}{8} & I & Work & Expert Client & Call reminders \\
\hline & II & Home & $\mathrm{CHW}$ & Travel vouchers \\
\hline & III & Neither & & \\
\hline
\end{tabular}

\section{8 choice sets. D-efficiency after iteration 0.9607}

\section{Data Analysis}

\section{Quantitative data}

We described participants' characteristics using descriptive summaries; frequency and percentages for categorical variables, median and inter-quartile range for count data like age, and duration on MDR-TB treatment.

\section{DCE analysis to determine the patients' preferred MDR-TB CB-DOT model}

For the primary analysis to determine patients' preferred attributes for community-based care, we used mixed logit choice model (with 1000 Halton simulation draws). We calculated utility scores by substituting coefficients into the model, and computed ranks to determine the most preferred MDR-TB CB-DOT attributes. We estimated the proportion of respondents that had positive relative preference for a certain attribute, using positive proportion = $\Phi(\beta / S D)$. Where, $\Phi=$ standard normal cumulative distribution function, and $\beta$ is positive coefficients from mixed logit choice model, and SD = standard deviation representing level of preference heterogeneity across participants. The following model was used:

Utility model $(V)=\beta 0+\beta 1 c_{-}$chw $+\beta 2$ c_expert $+\beta 3$ I_home $+\beta 4$ s_phcall $+\beta 5$ s_tvouch

Where,

V is utility derived from respondents' preference 
$\beta 1$ to $\beta 5$ are coefficients indicating relative importance of each attribute. ${ }^{15}$

For the secondary analysis to determine if these preferences differed by gender, age groups, HIV status, or duration on MDR-TB treatment, we fitted interactions to perform sub-group analysis. The significance level for all the analyses was determined at $5 \%$.

Qualitative data

A deductive approach with descriptive thematic coding was used to analyse data using NVivo Version 12. Interview transcripts were reviewed for content related to the research question. Codes were assigned to relevant segments of the text and similar codes aggregated to form themes that were used to develop coherent narratives. Data within and across themes were synthesized to generate an understanding of why certain attribute choices were preferred.

\section{Human subjects' approval}

Ethics approval was obtained from the University Research Co., LLC (URC) Ethics Review Board, the Joint Clinical Research Centre (JCRC) Ethics Review Board (Ref. JC1519), and the Uganda National Council of Science and Technology (UNCST) (Ref. HS2684). Prior to interview commencement, written informed consent was voluntarily obtained from all participants. Confidentiality of patients was ensured by using study identification numbers and data storage protection procedures.

\section{Results}

Socio-demographics

From December 2019 to January 2020, 103 participants were interviewed, characterized by the following socio-demographics: 58.3\% were male, the median age was 37 years (inter-quartile range [IQR] 30 to 47 year), and the majority were farmers (35.9\%), HIV negative (61.2\%), and earned less than $1 \$ /$ day $(65.1 \%)$. (Table 3). 
Table 3

Participants' Characteristics

\begin{tabular}{|c|c|}
\hline Characteristics & Number (\%), N = 103 \\
\hline \multicolumn{2}{|l|}{ Gender } \\
\hline Female & $43(41.7)$ \\
\hline Male & $60(58.3)$ \\
\hline Age in years, median (IQR) & $37(30-47)$ \\
\hline \multicolumn{2}{|l|}{ Age categories } \\
\hline $20-34$ & $42(40.8)$ \\
\hline $35+$ & $61(59.2)$ \\
\hline \multicolumn{2}{|l|}{ Marital status } \\
\hline Single/Single/Divorced/Separated & $48(46.6)$ \\
\hline Married & $55(53.4)$ \\
\hline \multicolumn{2}{|l|}{ Occupation } \\
\hline Farmer & $37(35.9)$ \\
\hline Business & $24(23.3)$ \\
\hline Employed & $33(32.1)$ \\
\hline Unemployed & $9(8.7)$ \\
\hline \multicolumn{2}{|l|}{ Daily income $(\$) \star$} \\
\hline$<1 \$$ & $67(65.1)$ \\
\hline$\geq 1 \$$ & $36(34.9)$ \\
\hline \multicolumn{2}{|l|}{ Educational level } \\
\hline None & $11(10.8)$ \\
\hline Primary & $58(56.9)$ \\
\hline Secondary & $24(23.5)$ \\
\hline Tertiary & $9(8.8)$ \\
\hline \multicolumn{2}{|l|}{ HIV status } \\
\hline Negative & $63(61.2)$ \\
\hline Positive & $40(38.8)$ \\
\hline \multicolumn{2}{|c|}{ Duration on MDR-TB treatment (months)* } \\
\hline Median (IQR) & $10(5-16)$ \\
\hline \multicolumn{2}{|l|}{ Categories } \\
\hline$<6$ months & $31(30.4)$ \\
\hline$\geq 6$ months & $71(69.6)$ \\
\hline
\end{tabular}

Discrete choice model analysis

All attributes were significant determinants of participants' preference of the attributes of community-based MDR-TB care. All coefficients had significant P-values $(<0.05)$ (Table 4) and a positive constant term (coefficient $=0.52$ ) implying that participants preferred at least one of the suggested hypothetical MDR-TB community-based care models to none.

\section{Preference for specific attributes of community-based care}

For specific attributes, the positive coefficients indicate that participants preferred: (1) treatment delivered by CHWs or expert clients rather than a family member; (2) treatment delivered at home rather than workplace; and (3) monthly travel vouchers as the form of additional support rather than phone call or SMS reminders. The estimated proportion of respondents with positive preference for CHWs and expert clients as the principal care giver for MDR-TB treatment, compared to a family member, was $73.0 \%$ and $69.2 \%$, respectively. The proportion of patients with positive preference for delivery of treatment 
at home compared to delivery at workplace was $85.4 \%$, whereas patients with positive preference for phone call reminders and monthly travel vouchers compared to SMS reminders was $99.9 \%$ and $99.3 \%$, respectively (Table 4).

Preference for models (combination of attributes) of community-based care

The most preferred community-based care model (i.e., combination of attributes) was a CHW giving treatment at the patient's home and the patient receiving monthly travel vouchers for additional support. The top four preferred MDR-TB CB-DOT models contained travel vouchers or phone call reminders and treatment delivered at home (Table 5).

\section{Sub-group analysis}

Sub-group analysis showed that patients' preference of the CB-DOT model attributes did not differ by gender. More HIV-positive than HIV-negative people with MDR-TB preferred their treatment delivered at home rather than at work (interaction term P-value $=0.04$ ). Older patients aged 35 years and above preferred a CHW more than younger patients (interaction term P-value $=0.02)$. Those who had been on MDR-TB treatment for a longer duration $(6$ months or more) preferred a CHW (interaction term P-value $=0.02$ ) or expert client (interaction term P-value $=0.02$ ).

Table 4

Mixed logit choice model estimates and standard deviations with percentage of positive preference for the MDR-TB CB-DOT model attributes

\begin{tabular}{|llllll|}
\hline Attributes & Coefficients & $95 \% \mathrm{Cl}$ & P-value & SD (SE) & \%Positive preference \\
\hline Constant (CBDOT model) & 0.52 & $0.30-0.74$ & $<0.01$ & - & - \\
\hline Model of delivery & & & & & \\
\hline Family & Reference & & & & \\
\hline Community health work & 1.13 & $0.68-1.59$ & $<0.01$ & $1.85(0.27)$ & 73.0 \\
\hline Expert client & 0.89 & $0.46-1.32$ & $<0.01$ & $1.77(0.27)$ & 69.2 \\
\hline Location & & & & & \\
\hline Workplace & Reference & & & & \\
\hline Home & 1.25 & $0.91-1.59$ & $<0.01$ & $1.19(0.20)$ & 85.4 \\
\hline Support & & & & & \\
\hline SMS & Reference & & & & \\
\hline Phone call reminders & 0.70 & $0.41-0.99$ & $<0.01$ & $0.19(0.58)$ & 99.9 \\
\hline Travel vouchers & 1.35 & $1.04-1.653$ & $<0.01$ & $0.55(0.26)$ & 99.3 \\
\hline
\end{tabular}

Mix logit choice model with continuous assumption of choices. $\mathrm{Cl}$ - Confidence interval, SD heterogeneity. - standard deviation for preference heterogeneity, SE - standard error. \% positive indicates proportion of patients with positive preference for an attribute. 
Table 5

Hypothetical MDR-TB CB DOT models ranks and utility scores

\begin{tabular}{|c|c|c|}
\hline Model & Utility score & Rank \\
\hline $\mathrm{H} 2 \mathrm{CHW} /$ Treatment delivered at home/Travel voucher & 4.25 & 1 \\
\hline F2 Expert clients/Treatment delivered at home/Travel voucher & 4.01 & 2 \\
\hline G2 CHW/Treatment delivered at home/Phone call reminder & 3.61 & 3 \\
\hline A2 Expert client/Treatment delivered at home/Phone call reminder & 3.36 & 4 \\
\hline E1 Family member/Treatment delivered at home/Travel voucher & 3.12 & 5 \\
\hline A1 CHW/Treatment delivered at work/Monthly travel voucher for monthly appointments & 3.00 & 6 \\
\hline E2 CHW/Treatment delivered at home/SMS reminder for monthly appointments & 2.90 & 7 \\
\hline B2 Expert client/Treatment delivered at work/Monthly travel voucher for monthly appointments & 2.76 & 8 \\
\hline D1 Expert client/Treatment delivered at home/SMS reminder for monthly appointments & 2.66 & 9 \\
\hline D2 Family member/Treatment delivered at home/Phone call reminder for monthly appointments & 2.48 & 10 \\
\hline $\mathrm{C} 2 \mathrm{CHW} /$ Treatment delivered at work/Phone call reminder for monthly appointments & 2.36 & 11 \\
\hline H1 Expert client/Treatment delivered at work/Phone call reminder for monthly appointments & 2.11 & 12 \\
\hline C1 Family member/Treatment delivered at home/SMS reminder for monthly appointments & 1.77 & 13 \\
\hline B1 Family member/Treatment delivered at home/SMS reminders for monthly appointments & 1.77 & 13 \\
\hline C1 Family member/Treatment delivered at home/SMS reminder for monthly appointments & 1.66 & 15 \\
\hline F1 CHW/Treatment delivered at work/SMS reminder for monthly appointments & 1.41 & 16 \\
\hline
\end{tabular}

\section{Qualitative results}

Several key themes emerged from the data regarding the preferred attributes of community-based model of care.

\section{Provider Type}

Respondents reported that a CHW or expert client was preferred because they were trained and knowledgeable. In addition, they had the ability to offer adherence and psychosocial support including guidance on how to take their medications because of their professional or personal experience. Further, patients felt that expert clients could be more empathetic because they have been through similar experiences. They therefore trusted them to maintain confidentiality in the process of offering care.

"A community health worker encourages you to take medicine than any other person. Also provides counselling to the patient and explains the benefit of taking medicines on time." [20 year., Female, Single]

"I prefer an expert client to support me since he has been through the same situation. Expert client has the best experience ever so [I] will be able to share with him." [33 year., Male, Married]

"I prefer a CHW because they are knowledgeable, and I can trust them with my life." [54 year., Male, Married]

Family members were least preferred and viewed as unable to offer the support needed.

"......... family members, they sympathize so much and may discourage you from sticking to your treatment schedules as required and also they lack experience with this type of TB disease." [22 year., Female, Single]

\section{Location of Care}

Patients preferred to receive care from home citing privacy and a lower risk of TB-related stigma. They also felt that receiving care at home saves them time and money and presents an opportunity for health education for their family.

"The treatment at home also is a bonus, because the community cannot discriminate if confidentiality is kept by the expert client." [29 year., Female, Divorced/separated]

Treatment at home and monthly travel vouchers for monthly appointments is my preferred choice because there is time management while taking drugs since no out movement.

[66 year., Male, Married] 
"It becomes less costly when I get treatment at home." [31 year., Male, Married]

The workplace was not preferred by most of the respondents. It was associated with stigma and fear for loss of employment or clients.

"I feel confidentiality is kept if treatment is given at home compared to my workplace where / could easily be seen and get stigmatized." [22 year., Female, Divorced/separated]

"At home there is no stigma on my condition and yet if visited at work, I could easily suffer from the gossip by workmates when they eventually learn about my condition, this could easily make me lose clients." [55 year., Female, Divorced/separated]

\section{Support Type}

Participants felt that monthly travel vouchers were the best way to promote appointment keeping as they provided relief from worrying about transport costs and money left over transport fares could be used to meet other needs like food, other household items, payment of debts, savings and farming amongst others.

"Monthly travel vouchers are the best for me because I will be knowing that transport is readily available and even the ticket will be reminding me of the clinic appointment date." [40 year., Male, Married]

"Because the money given for the travel voucher, the balance from it I can use it to buy other things and also do farming with it." [52 year., Male, Married]

Some of the respondents did not prefer other types of patient support, e.g., SMS or phone calls, due to various limitations surrounding their use which included:

"The message (SMS) reminders would work for me since am partially deaf and cannot easily hear through a phone call......" [43 year., Female, Divorced/separated]

".......SMS reminders are not good for me because I don't know how to read but may be if they call me and give transport to the clinic." [40 year., Male, Married]

\section{Discussion}

We carried out a parallel convergent mixed methods study to determine patient preferences for different attributes of community-based care. We found that people with MDR-TB preferred care to be provided at home by a CHW or an expert client who is a member of the community and as additional support, to receive travel vouchers to enable attendance at monthly clinic follow-up visits. MDR-TB patients preferred community-based care to the current standard of care, which is HF-DOT. CHWs and expert clients were viewed as knowledgeable, experienced, empathetic, and skilled to properly counsel and guide patients on how to manage side effects. They were also seen as able to maintain confidentiality in the process of offering care. Family members were viewed as lacking adequate MDR-TB related knowledge and patients were skeptical of their ability to offer the support required.

Preference for and patient satisfaction with lay providers has also been observed elsewhere, such as in rural Swaziland where DOT and administration of injectable forms of MDR-TB medication was delegated to trained community treatment supporters. ${ }^{16}$ In that study, preference for lay providers was driven by their ability to offer adherence counselling, confidentiality, and perceived lower treatment costs, reasons similar to those given by patients in our study.

Most patients preferred home care noting it provides privacy, safety, and comfort. Further, patients viewed home-based care as being less costly as it saves on time spent accessing care and daily transportation costs to follow-up health facilities. The workplace was perceived as a possible source of stigma that could lead to loss of clientele for small business owners or loss of employment. Similar to our study, findings from rural northern Uganda ${ }^{17}$ showed that home-based care was acceptable to both patients and health providers noting that it is safe, conducive to recovery and time saving. This study further showed that home-based care enabled psychosocial support. In our study, however, psychosocial support was mentioned as a reason for preference for a certain provider type rather than place of care.

In Bangladesh, a decentralized, community-based treatment program for patients with drug-resistant tuberculosis used home-based care DOT to address various needs of MDR-TB patients. It was a patient's preferred approach evidenced by their retention in care resulting into improvement in other treatment outcomes. ${ }^{18}$ Similarly, a quasi-experimental study done in India showed home-based care to be associated with low stigma ${ }^{19}$ similar to our study findings. In rural South Africa, MDR-TB patients preferred to receive MDR-TB and HIV care at home, and this was associated with reduced levels of rejection creating strong emotional bonds between patients, families and communities that is critical to health. ${ }^{20}$ The home is seen as a place conducive for recovery and offers both psychological and emotional support needed to enable healing. ${ }^{21}$ In our study, patients preferred to receive care at home noting that home is private, is associated with low levels of stigma, saves time and money, and provides an opportunity for health education including infection prevention and control at the family level. Specifically, patients who were MDR-TB HIV co-infected preferred to be treated from home than those who were HIV negative. This may be related to the fact that in 2017, Uganda rolled out differentiated service delivery models for persons infected with HIV that included community-based drug delivery options. ${ }^{22}$ It is possible that clients' positive experience with these care delivery models positively influenced their choice for home based care. ${ }^{23}$ 
Despite documented evidence that digital mobile technologies are useful in supporting TB care, ${ }^{24}$ majority of participants preferred monthly travel vouchers over mobile-based support, such as SMS and phone call reminders. In our study, varying degrees of literacy and hearing loss due to drug toxicity probably decreased the preference for mobile technologies. In addition, the utility of the travel voucher in meeting other household needs besides travel to the monthly hospital appointment served as a main driver for this choice. The majority of our respondents earned only about a dollar a day and were receiving a transport voucher worth about 50 dollars a month from the NTLP under its ongoing "enabler program". The travel voucher therefore shielded them against incurring catastrophic costs during their treatment. In Uganda, a recent study done to examine costs incurred during TB treatment showed that that more than half of households affected by TB experienced catastrophic costs, defined as spending more than $20 \%$ of their annual income on TB and these costs were 30 times higher among MDR-TB patients compared to drug sensitive tuberculosis (DS-TB) patients. ${ }^{25}$ The major drivers of cost according to the Uganda TB cost survey were non-medical and included transportation, food, and nutritional supplements. Many respondents in our study reported that they could use the travel voucher to buy food.

The study had several strengths and limitations. We had regional representation across the country, involved MDR-TB treatment centers that treat more than $80 \%$ of the MDR-TB cases. The results presented here are therefore applicable to MDR TB patients diagnosed across the country. In addition, we used patients who had been on treatment for MDR-TB. Their opinions therefore are grounded in their lived experiences. However, the retrospective nature of the study may have resulted in some recall bias which could have resulted in under reporting. However, we tried to minimize this by using hospital records for non DCE variables. In addition, DCE experiments are sometimes prone to DCE bias which could result in misrepresentation of preferences. This was minimized by using pictorial questionnaires to aide understanding of choice sets. Further, the study used routinely collected data which is prone to missing data. However, efforts were made to minimize this by training research assistants on quality data collection and respective standard operating procedures prior to data collection. Finally, data collected did not include children and other risk populations, like pregnant women. Future studies could include children and other vulnerable populations so that their views are taken into consideration.

\section{Conclusions And Recommendations}

Our respondents preferred to take their medicines at home supported by a member of their community but revealed a critical need for additional support to help mitigate the costs of accessing care. Due to the current COVID-19 pandemic, innovations that reduce the need for health facility visits while still providing additional support to meet client needs will become increasingly relevant. ${ }^{2}$ The feasibility and effectiveness of these models of care should be further evaluated. Studies to determine the feasibility and effectiveness of our preferred patient care model are underway.

\section{Declarations}

\section{ETHICS APPROVAL AND CONSENT TO PARTICIPATE}

This research was performed in accordance with the Declaration of Helsinki guidelines. It was approved by the Joint Clinical Research Centre (JC1519) Institutional Review Board (IRB), and by the Uganda National Council of Science and Technology (HS2684) prior to study conduct. Administrative permission to collect this data were provided by the National TB and Leprosy Program. Prior to interview commencement, written informed consent was voluntarily obtained from all participants. Confidentiality of patients was ensured by using study identification numbers and data storage protection procedures.

\section{AUTHOR CONTRIBUTIONS}

RMM- Study conceptualization, protocol development, supervision of data collection process, qualitative data analysis, preparation of initial draft

JM- Protocol review, quantitative data analysis, manuscript revisions

SZM- Study conceptualization, protocol review, supervision of data collection process, manuscript revisions

EK: protocol review, supervision of data collection process, MDR TB technical input, manuscript revisions

HN: Supervision of data collection process, data management, manuscript revisions

PA: Supervision of data collection process, data management, manuscript revisions

DC- Protocol review, manuscript revisions, review of qualitative data analysis output, overall study supervision

DBFL- Protocol review, manuscript revisions, overall study supervision

HB- Protocol review, qualitative data analysis, manuscript revisions

ST- Study conceptualization, protocol review, overall study supervision, manuscript revisions.

AN- Study conceptualization, protocol review, overall study supervision, manuscript revisions. 
The authors are indebted to MDR-TB patients in Uganda who participated in the study. We thank the administration and health workers of the hospitals where this study was carried out for their invaluable help during the data collection process. We appreciate the input and guidance of health authorities, the Ministry of Health-NTLP, Uganda. Many thanks to Makerere University Implementation Science Program for the invaluable input especially during protocol development

\section{FUNDING}

Support for the conduct of this study was provided by USAID through the Defeat TB Project. USAID Cooperative Agreement Number AID-617-A-17-00003.

\section{AVAILABILITY OF DATA AND MATERIALS}

The datasets used and/or analyzed during the current study available from the corresponding author on request.

\section{CONSENT FOR PUBLICATION}

Not applicable

\section{COMPETING INTERESTS}

The authors declare that they have no competing interests.

\section{References}

1. World Health Organization. WHO treatment guidelines for drug-resistant tuberculosis: 2016 update. https://www.who.int/publications/i/item/9789241549639

2. Global Tuberculosis Report 2020. https://www.who.int/publications/i/item/9789240013131

3. Uganda Ministry of Health. NTLP Annual Report, September 2019.

4. Jia Yin, Jinqiu Yuan, Yanhong Hu XW. Association between Directly Observed Therapy and Treatment Outcomes in Multidrug-Resistant Tuberculosis_ A Systematic Review and Meta-Analysis. PLoS One. 2016;1-14.

5. Uganda Ministry of Health. Uganda National Guidelines for Programmatic Management of Drug-Resistant Tuberculosis. 2016. p. 300.

6. Ministry of Health Kenya. The First Kenya Tuberculosis Patient Cost Survey. 2017.

7. Loveday, Marian. Wallengren, Kristina. Brust, J. Roberts, J. Voce, A. Margot, B. Ngozo, J. Master, L. Cassell, G. Pdayatchi N. NIH Public Access. Int J Tuberc Lung Dis. 2015;19(2):163-71.

8. Floyd K, Wilkinson D, Gilks C. Comparison of cost effectiveness of directly observed treatment (DOT) and conventionally delivered treatment for tuberculosis: experience from rural South Africa

9. E. S, K. F, D. M, L. D, R. G, V. A. Cost and cost-effectiveness of community-based care for tuberculosis in Cape Town, South Africa. Int J Tuberc Lung Dis [Internet]. 2003;7(9): S56-62.

10. Khan MA, Walley JD, Witter SN, Imran A, Safdar N. Costs and cost-effectiveness of different DOT strategies for the treatment of tuberculosis in Pakistan. Directly Observed Treatment. Health Policy Plan. 2002 Jun;17(2):178 - 86. doi: 10.1093/heapol/17.2.178. PMID: 12000778.

11. WHO. Rapid Communication: Key changes to treatment of multidrug- and rifampicin-resistant tuberculosis. World Heal Organ. 2018;(August): License: CC BY-NC-SA 3.0 IGO.

12. Johnson FR, Lancsar E, Marshall D, Kilambi V, Mühlbacher A, Regier DA, et al. Constructing experimental designs for discrete-choice experiments: report of the ISPOR conjoint analysis experimental design good research practices task force. Value Health. 2013;16(1):3-13. pmid:23337210

13. Bridges JF, Hauber AB, Marshall D, Lloyd A, Prosser LA, Regier DA, et al. Conjoint analysis applications in health-a checklist: a report of the ISPOR Good Research Practices for Conjoint Analysis Task Force. Value Health. 2011;14(4):403-13. pmid:21669364

14. Wong SF, Norman R, Dunning TL, Ashley DM, Lorgelly PK. A protocol for a discrete choice experiment: understanding preferences of patients with cancer towards their cancer care across metropolitan and rural regions in Australia. BMJ Open. 2014 Oct 24;4(10):e006661. doi: 10.1136/bmjopen2014-006661.

15. Hauber AB, Gonzalez JM, Groothuis-Oudshoorn CG, Prior T, Marshall DA, Cunningham C, et al. Statistical Methods for the Analysis of Discrete Choice Experiments: A Report of the ISPOR Conjoint Analysis Good Research Practices Task Force. Value Health. 2016;19(4):300-15. pmid:27325321

16. Peresu E, Heunis CJ, Kigozi GN, De Grave D. Patient satisfaction with directly observed treatment and multidrug-resistant tuberculosis injection administration by lay health workers in rural Eswatini. Afr J Prim Health Care Fam Med. 2020 May 26;12(1):e1-e10. doi: 10.4102/phcfm.v12i1.2257. PMID: 32501027; PMCID: PMC7284164.

17. Horter S, Stringer B, Reynolds L, Shoaib M, Kasozi S, Casas EC, Verputten M, du Cros P. "Home is where the patient is": a qualitative analysis of a patient-centred model of care for multi-drug resistant tuberculosis. BMC Health Serv Res. 2014 Feb 21;14:81. doi: 10.1186/1472-6963-14-81. PMID: $24559177 ;$ PMCID: PMC3943511.

18. Daru P, Matji R, AlMossawi HJ, Chakraborty K, Kak N. Decentralized, Community-Based Treatment for Drug-Resistant Tuberculosis: Bangladesh Program Experience. Glob Health Sci Pract. 2018 Oct 4;6(3):594-602. doi: /GHSP-D-17-00345. Erratum in: Glob Health Sci Pract. 2018 Dec 27;6(4):766. PMID: 30287534; PMCID: PMC6172109. 
19. Taneja N, Chellaiyan VG, Daral S, Adhikary M, Das TK. Home Based Care as an Approach to Improve the Efficiency of treatment for MDR Tuberculosis: A Quasi-Experimental Pilot Study. J Clin Diagn Res. 2017 Aug;11(8):LC05-LC08. doi: 10.7860/JCDR/2017/27594.10401. Epub 2017 Aug 1. PMID: $28969162 ;$ PMCID: PMC5620803.

20. Brust JC, Shah NS, Scott M, Chaiyachati K, Lygizos M, van der Merwe TL, Bamber S, Radebe Z, Loveday M, Moll AP, Margot B, Lalloo UG, Friedland GH, Gandhi NR. Integrated, home-based treatment for MDR-TB and HIV in rural South Africa: an alternate model of care. Int J Tuberc Lung Dis. 2012 Aug;16(8):998-1004. doi: 10.5588/ijtld.11.0713. Epub 2012 Jun 5. PMID: 22668560; PMCID: PMC3390442.

21. 27. Adatu F, Odeke R, Mugenyi M, Gargioni G, McCray E, Schneider E, Maher D. Implementation of the DOTS strategy for tuberculosis control in rural Kiboga District, Uganda, offering patients the option of treatment supervision in the community, 1998-1999. Int J Tuberc Lung Dis. 2003 Sep;7(9 Suppl 1):S63-71. PMID: 12971656

22. Implementation Guide for Differentiated Service Delivery Models of HIV Services in Uganda, June 2017. Accessed at https://www.google.com/search?

q=26.+Implementation+Guide+for+Differentiated+Service+Delivery+Models+of+HIV+Services+in+Uganda\%2C+June+2017.\&rlz=1C1GCEU_enUG824U

23. Zakumumpa H, Bennett S, Ssengooba F. Modifications to ART service delivery models by health facilities in Uganda in promotion of intervention sustainability: a mixed methods study. Implement Sci. 2017 Apr 4;12(1):45. doi: 10.1186/s13012-017-0578-8. PMID: 28376834; PMCID: PMC5379666.

24. Lee Y, Raviglione MC, Flahault A. Use of Digital Technology to Enhance Tuberculosis Control: Scoping Review. J Med Internet Res. $2020 ; 22(2):$ e15727. Published 2020 Feb 13. doi:10.2196/15727

25. Muttamba, W., Tumwebaze, R., Mugenyi, L. et al. Households experiencing catastrophic costs due to tuberculosis in Uganda: magnitude and cost drivers. BMC Public Health 20, 1409 (2020). https://doi.org/10.1186/s12889-020-09524-5 